\title{
Large Vessel Occlusion Causing Cerebral Ischemic Stroke in Previously Healthy Middle-Aged Recently Recovered from Severe COVID-19 Infection
}

\author{
Ali Alhashim \\ Mustafa Alqarni \\ Majed Alabdali \\ Mohammed Alshurem \\ Aishah Albakr \\ Kawther Hadhiah (D) \\ Danah Aljaafari \\ Rawan Alyami
}

Neurology Department, College of Medicine, Imam Abdulrahman Bin Faisal University, Dammam, 34212, Saudi Arabia
Correspondence: Ali Alhashim Neurology Department, Medical College, Imam Abdulrahman Bin Faisal University, Dammam, 34212, Saudi Arabia Tel +966509444833

Email ahalhashem@iau.edu.sa; ali_al_hashem@hotmai.com
Background: Severe acute respiratory syndrome coronavirus (SARS-CoV-2) due to novel coronavirus disease 2019 (COVID-19) has led to an unprecedented worldwide pandemic with diverse respiratory symptoms as well as systemic manifestations and complications. The neurological manifestations of COVID-19 include, but are not limited to, headache, cerebrovascular disease, and skeletal muscle injury.

Case Report: Herein, we present a case of stroke with large vessel occlusion in a middleaged man, who recently recovered from severe SARS-CoV-2 infection. This patient is not known to have any medical illness or surgical history and has no cerebrovascular risk factors. Moreover, the patient underwent extensive investigations, including neuroimaging, cardiac and laboratory work-up with no evidence of stroke etiology.

Conclusion: The mechanism of cerebrovascular events in the setting of COVID-19 is still uncertain and probably multi-factorial. The prevailing hypothesis is a strong thrombotic tendency, which may even be prolonged after complete recovery. In our patient's case, hypercoagulability in the context of viral infection is the most likely mechanism for the stroke. Further studies are needed to find out the exact pathogenesis of thromboembolic events in the setting of COVID-19 infection as well as the efficacy, safety, dosage, and duration of anticoagulants in such conditions.

Keywords: large vessel occlusion, stroke, healthy middle-aged, viral hypercoagulability, SARS-CoV-2, independent risk factor

\section{Introduction}

Novel coronavirus (COVID-19) is responsible for an unprecedented global pandemic that has a significant impact on health systems worldwide. The clinical presentation of COVID-19 is widely variable. It varies from asymptomatic or mild respiratory symptoms to severe pneumonia with acute respiratory distress syndrome (ARDS) requiring intensive care unit (ICU) admission associated with high morbidity and mortality.,2 Furthermore, there is growing evidence of multisystemic involvement, which includes the central nervous system. The neurological manifestations of COVID-19 include, but are not limited to, headache, altered level of consciousness, cerebrovascular disease, and skeletal muscle injury. ${ }^{3}$ Herein, we report a case of cerebral large vessel occlusion in a healthy athletic middle-aged man, most likely due to hypercoagulability after he recovered from severe COVID-19 infection. We also briefly review COVID-19 related stroke literature. 


\section{Case Presentation}

We report a fifty-six-year-old male who has recently recovered from severe pneumonia due to SARS-CoV-2 infection. The diagnosis was then confirmed with a positive polymerase chain reaction (PCR) nasopharynx swab. Chest x-ray and chest computed tomography (CT) findings, along with the patient's clinical presentation, supported the diagnosis as well. The patient was treated and then discharged home after complete recovery with evidence of negative PCR.

Few days after discharge, he presented to our emergency department with acute-onset neurological deficit in the form of visual deficit and altered sensation of the left side of the body. Apart from the recent recovery of severe COVID-19 infection, there was no history suggestive of cardiovascular risk factors. Additionally, he is not taking any medications regularly. Family and social history were unremarkable. Substance use, including illicit drugs, alcohol, and nicotine was credibly denied.

Upon presentation, blood pressure was 143/85 mmHg, pulse rate was 83 beats per minute, and his temperature was $36.7^{\circ} \mathrm{C}$. Neurological examination revealed left complete homonymous hemianopia, dysarthria, left pronator drift, left upper extremity ataxia, and left sensory loss with a total National Institutes of Health Stroke Scale (NIHSS) of 8 points. Since the patient presented within the thrombolysis window for acute stroke (around 3:45 hours from symptoms onset), Stroke-Code was activated. Accordingly, urgent cerebral CT and CT-angiography (Figure 1) were done, which showed a proximal occlusion of the right posterior cerebral artery (PCA) at the P1-P2 junction without signs of established acute ischemic stroke or hemorrhage.

After ruling out all other contraindications of thrombolysis, the patient received systemic intravenous thrombolytic therapy. Furthermore, we decided to proceed with mechanical thrombectomy as the thrombus was located proximally, and patient-related factors were favorable; these include age, absence of other medical comorbidities, and prior good functional status (Modified Rankin Scale "mRS": 0). Thrombolysis in cerebral infarction (TICI) $2 \mathrm{~b}$ was achieved as shown in (Figure 2). Despite the timely intervention, there was no clinical improvement after recanalization therapies. The follow-up CT (Figure 3) revealed an acute large ischemic stroke in the whole territory of a right posterior cerebral artery (PCA) up to the thalamus with substantial hemorrhagic transformation in the shape of parenchymal hematoma type 1 (PH1). The patient was also taken for a magnetic resonance imaging (MRI) scan, which did not reveal any new findings (Figure 4).

To classify the etiology of the stroke, several investigations have been obtained including transthoracic and transesophageal echocardiogram as well as long-term electrocardiography (ECG) monitoring for 72 hours,

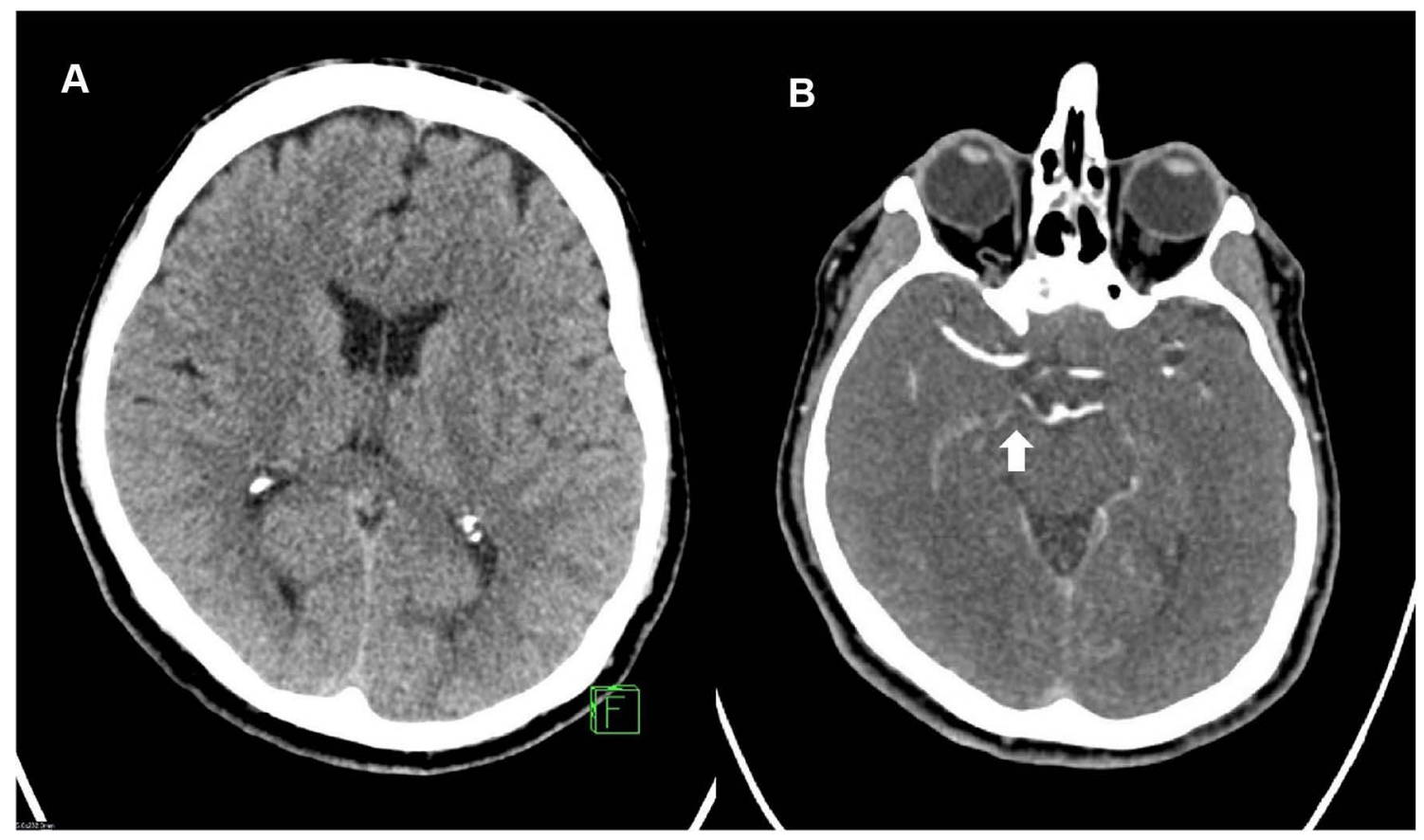

Figure I Non contrasted CT (A) and CT-Angiography (B) show a proximal occlusion of the right posterior cerebral artery (PCA) at PI-P2 junction (arrow) without signs for acute ischemic stroke or hemorrhage. 


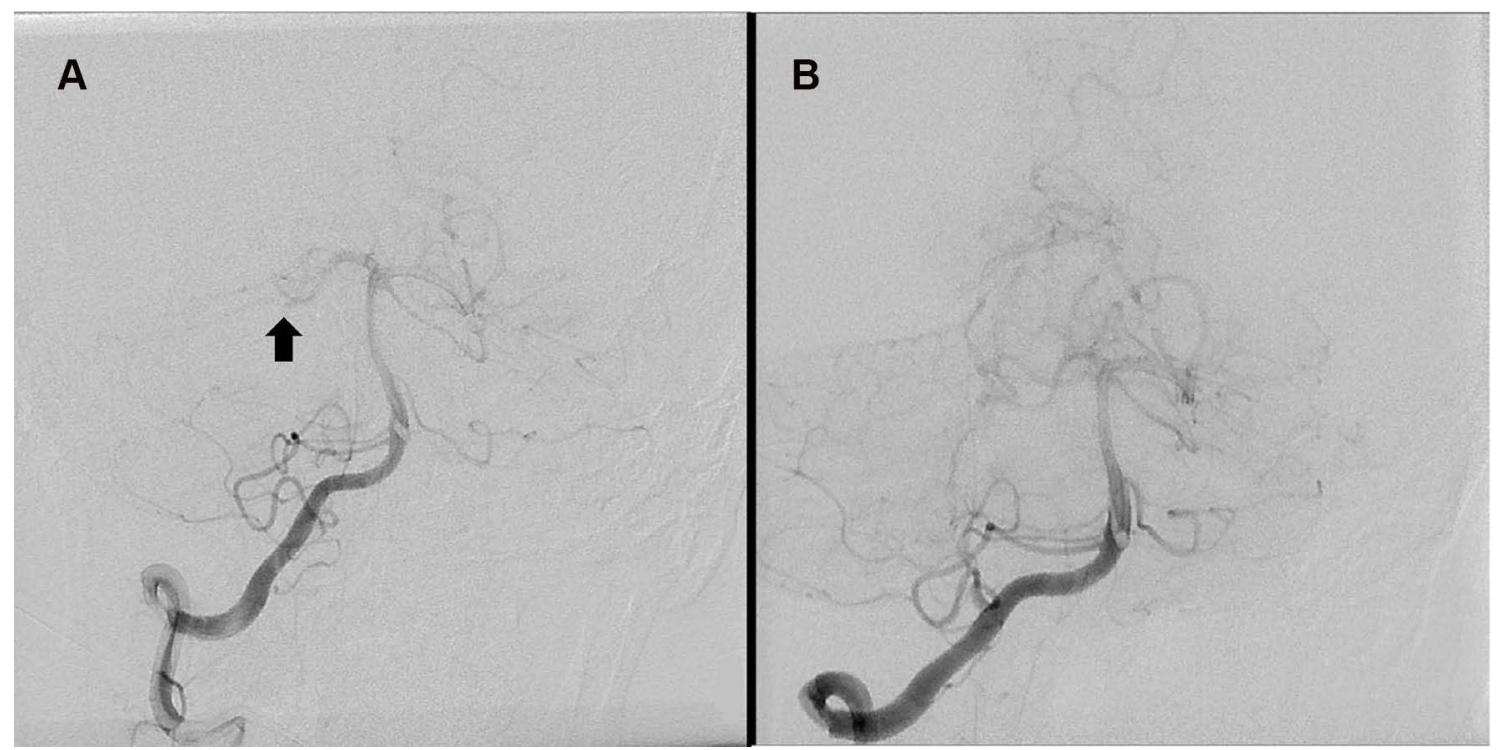

Figure 2 Digital subtraction angiography $(\mathbf{A})$ before thrombectomy shows right PI-P2 occlusion (arrow), and (B) after thrombectomy shows near complete perfusion with $\mathrm{TICl} 2 \mathrm{~b}$.

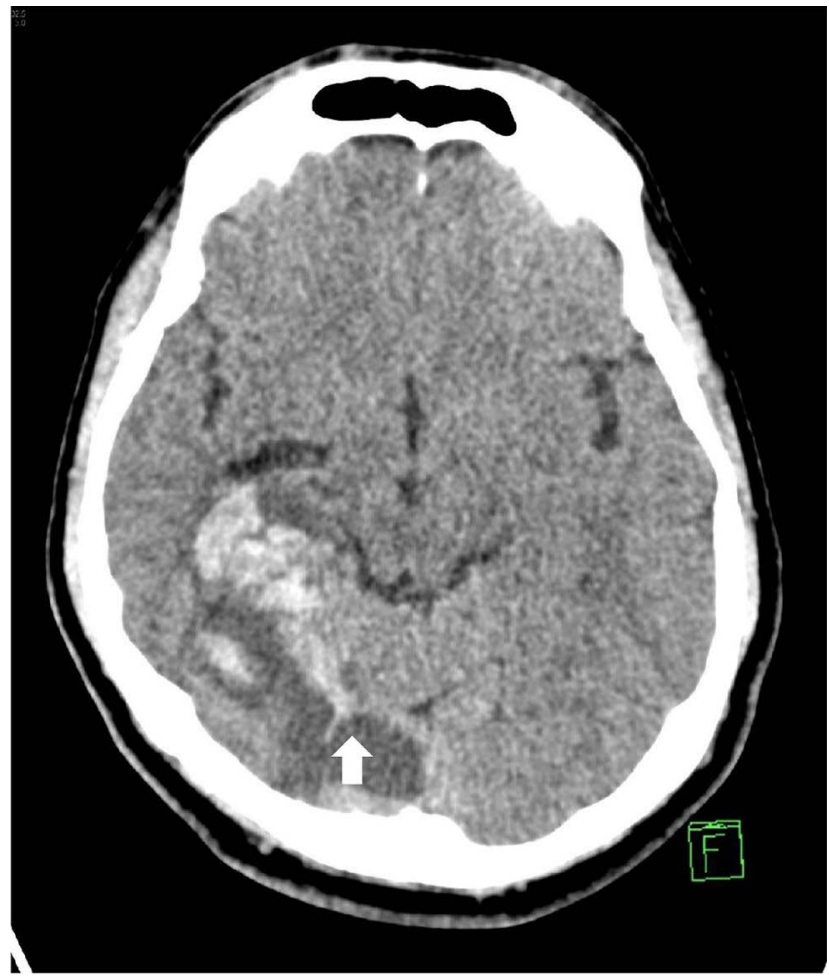

Figure 3 CT follow up 24 hours after acute reperfusion therapies shows acute ischemic stroke in right PCA territory with substantial hemorrhagic transformation (arrow).

which could not detect any evidence of cardiogenic embolic sources. In particular, there was no evidence of atrial fibrillation, regional wall motion abnormalities, patent foramen ovale (PFO), atrial septal anomalies, or valvular dysfunction. Additionally, the long-term ECG monitoring was repeated in the outpatient department, and it did not reveal any arrhythmias. The cerebral CTangiography of the head and neck showed no evidence of atherosclerotic macroangiopathy or source of an artery-toartery embolism, besides the right PCA occlusion. Laboratory investigations showed elevation of anticardiolipin IgG, plasma D-dimer, erythrocyte sedimentation rate (ESR), and borderline antinuclear antibodies titer (ANA), which reflects a persistent prothrombotic state even after clinical recovery of COVID-19 infection (Table 1). HbA1c was normal, while low-density lipoprotein (LDL) was mildly elevated. The thrombophilia screening tests were negative.

\section{Discussion}

There is growing evidence that suggests the relation between COVID-19 and increased risk of arterial as well as venous thromboembolic complications, including acute ischemic stroke, myocardial infarction, deep vein thrombosis, and pulmonary embolism. ${ }^{4-9}$ This case sheds light on some possible mechanisms of COVID-19 related stroke. The exact mechanism of COVID-19 causing strokes is still uncertain. Several mechanisms are proposed including a hypercoagulable state ${ }^{8-10}$ and vasculitis due to vascular endothelial cell infection with SARS-CoV-2 leading to endotheliitis. ${ }^{11}$ It seems likely that anticoagulants will play an important role in the prevention of stroke in COVID-19 patients. This suggestion is based on small case series, prospective, as well as retrospective 

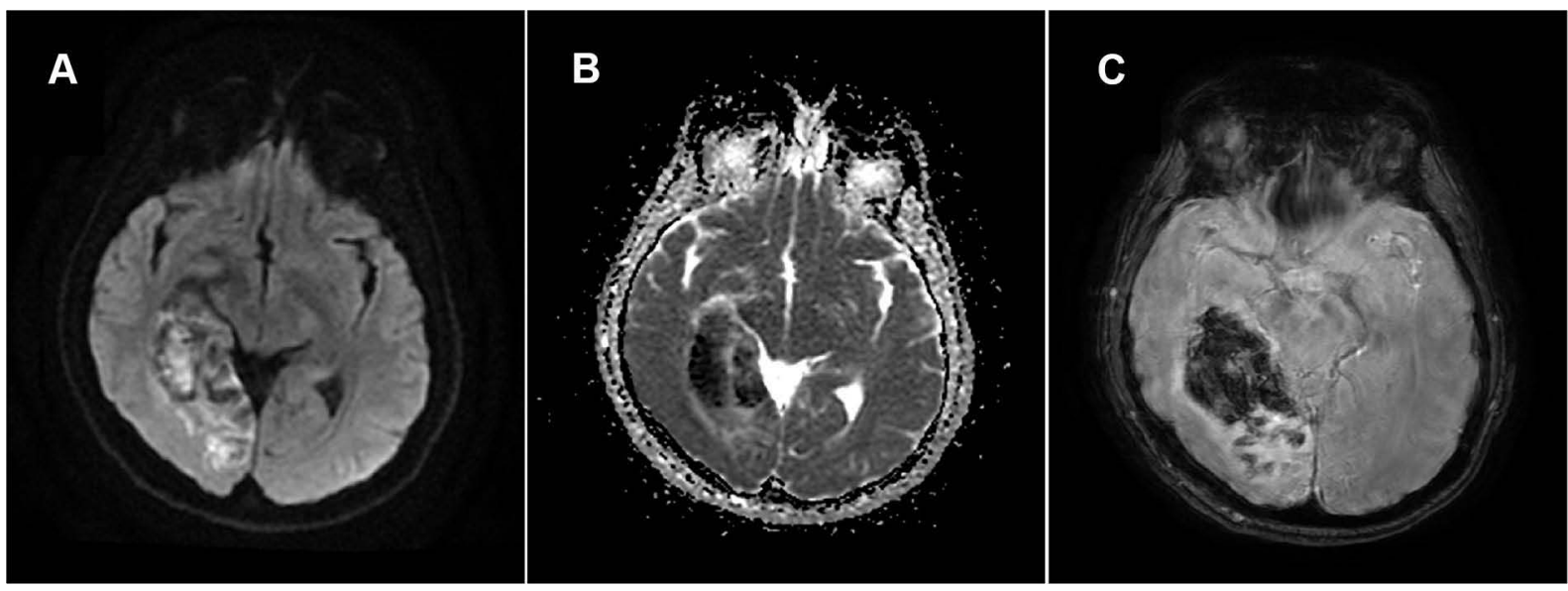

Figure 4 Axial brain MRI images of diffusion-weighted imaging (A), apparent diffusion coefficient (B), and susceptibility-weighted imaging (C) sequences, show acute ischemic changes in the right PCA territory with hemorrhagic transformation.

observational studies; therefore, further evidence is needed from larger-scale studies to confirm this theory.

To our knowledge, most of the reported cases in the literature of COVID-19 patients with large vessel occlusion were elderly and had known cardiovascular risk factors. ${ }^{9}$ However, our patient is a healthy middle-aged man with no relevant cerebrovascular risk factors. This observation is unique and supports the suggestion that SARS-COV-2 infection could be an independent risk factor for ischemic stroke. ${ }^{5}$ Furthermore, most of the reported events of large vessel occlusion were at the early phase of COVID-19 infection ${ }^{4,8,12}$ or during the course of the disease, ${ }^{13}$ unlike our patient who developed a stroke after a recent complete recovery of COVID-19 infection. This finding is unique too and goes along with a case reported by Mowla et al. ${ }^{14}$ These two cases suggest the possibility of a prolonged prothrombotic state even after recovery from COVID-19.

Another possible mechanism of stroke post-COVID-19 infection is the development of vasculitis due to endotheliitis. ${ }^{11,15}$ However, in our case, there were no conclusive findings, neither clinical nor laboratory or radiological that denote vasculitis.

Despite patient presentation within the thrombolysis window and timely intervention, the outcome of acute management was disappointing, as the patient did not improve clinically, and the follow-up neuroimaging showed complete PCA infarction with significant hemorrhagic transformation. Similarly, hemorrhagic transformation following ischemic stroke in patients with active SARS-CoV-2 infection who do not have cardiovascular risk factors has been also reported; however, these patients did not receive thrombolytic therapy since the transformation was detected in the initial neuroimaging. ${ }^{16}$ This could be explained by the fact that patients with COVID-19 have a significantly higher incidence of hemorrhagic transformation compared with their non-infected counterparts. ${ }^{17}$ Although the hemorrhagic transformation might be a potential complication of thrombolytic therapy, it is unlikely since the patient was eligible for the treatment and did not have any risk factors for bleeding.

Regarding our patient's poor outcome, a study conducted by Cappellari et al found that stroke patients with concurrent SARS-CoV-2 infection have a worse outcome compared with previous studies of patients having strokes alone. This is true for both patients who received intravenous thrombolysis alone and those who received it as a bridging therapy before undergoing thrombectomy. ${ }^{18}$ Additionally, a study has shown that having a poor outcome after receiving reperfusion therapies was independently associated with SARS-CoV-2 infection. ${ }^{19}$ This finding is in agreement with another study done by Escalard et al, describing that poor outcome could be related to the prothrombotic state due to SARS-CoV-2 infection causing re-occlusions after receiving reperfusion therapies. ${ }^{20}$

\section{Conclusion}

This case adds to the growing body of evidence that confirms the relation between cerebrovascular thromboembolic event and COVID-19 even in the absence of typical cardiovascular risk factors and supports that SARS-COV-2 infection could be an independent risk factor for ischemic stroke. 
Table I Results of Laboratory Tests Performed on Admission

\begin{tabular}{|c|c|c|}
\hline Test & Result & Reference Range \\
\hline \multicolumn{3}{|l|}{ Hematology } \\
\hline RBC (Mil/ul) & 4.7 & $4.7-6.1$ \\
\hline WBC (k/ul) & 9.0 & $4.1-11.0$ \\
\hline Segs (\%) & 79.8 & $40-75$ \\
\hline Lymphocyte (\%) & 10.1 & $20-45$ \\
\hline Hemoglobin (g/dl) & 13.6 & $13.0-18.0$ \\
\hline Platelets (k/ul) & 341 & $140-450$ \\
\hline \multicolumn{3}{|l|}{ Blood chemistry } \\
\hline Random blood sugar (mg/dl) & 107 & $70-140$ \\
\hline Hemoglobin AIC (\%) & 5.9 & $<6 \%$ \\
\hline Total cholesterol (mg/dl) & 190 & $<200$ \\
\hline LDL (mg/dl) & 129 & $<100$ \\
\hline Troponin (ng/mL) & 0.002 & $<0.02$ \\
\hline \multicolumn{3}{|l|}{ Coagulation } \\
\hline PT $(\mathrm{Sec})$ & 11.2 & $10.0-14.0$ \\
\hline aPTT (Sec) & 23.7 & $20.0-40.0$ \\
\hline INR & 1.02 & \\
\hline Plasma D-dimer (ug/mL) & 18.72 & $<0.5$ \\
\hline \multicolumn{3}{|l|}{ Inflammatory markers } \\
\hline ESR $(\mathrm{mm} / \mathrm{hr})$ & 37 & $0-20$ \\
\hline Quantitative CRP (mg/dl) & $<0.1$ & $0.1-0.5$ \\
\hline \multicolumn{3}{|l|}{ Autoimmune work-up } \\
\hline Antinuclear antibodies (ANA) & $\mathrm{I}: 80$ & $<1: 80$ \\
\hline Anti-double strand DNA & $<1: 10$ & $<1: 10$ \\
\hline Lupus anticoagulant screen ratio & 0.94 & $<1.2$ \\
\hline Anti- cardiolipin IGM & $<1.0$ & $<20$ \\
\hline Anti- cardiolipin IgG & 28.0 & $<20$ \\
\hline
\end{tabular}

Abbreviations: RBC, red blood cell; WBC, white blood cell; LDL, low density lipoprotein; aPTT, activated partial thromboplastin time; PT, prothrombin time; INR, international normalized ratio; Sec, Second; DNA, deoxyribonucleic acid; IgG, immunoglobulin G; IgM, immunoglobulin M; ESR, erythrocyte sedimentation rate; CRP, C-reactive protein.

Further studies are needed to find out the exact pathogenesis of thromboembolic events and to investigate the efficacy, safety, as well as the exact dose, and the duration of anticoagulation for the prevention of thromboembolic complications in the setting of COVID-19 infection.

\section{Abbreviations}

SARS-CoV-2, severe acute respiratory syndrome coronavirus; ARDS, acute respiratory distress syndrome; ICU, intensive care unit; PCR, polymerase chain reaction; CT, computed tomography; PCA, posterior cerebral artery; TICI, thrombolysis in cerebral infarction; PH1, parenchymal hematoma type 1; PFO, patent foramen ovale; MRI, magnetic resonance imaging; ESR, erythrocyte sedimentation rate; ANA, antinuclear antibodies titer; LDL, low-density lipoprotein; IRB, institutional review board.

\section{Statement of Ethics}

The subject has provided his informed consent for the creation of this manuscript with full knowledge that his clinical findings and treatment are utilized herein for educational and peer-reviewed publication purposes.

\section{Consent for Publication}

Written informed consent was obtained from the patient for publication of this case report and any accompanying images. Approval from the IRB committee at Imam Abdulrahman Bin Faisal University was not required for publishing these case details.

\section{Disclosure}

The authors report no conflicts of interest in this work.

\section{References}

1. Lai CC, Liu YH, Wang CY, et al. Asymptomatic carrier state, acute respiratory disease, and pneumonia due to severe acute respiratory syndrome coronavirus 2 (SARS-CoV 2): facts and myths. $J$ Microbiol Immunol Infect. 2020;53:404e412. doi:10.1016/j. jmii.2020.02.012

2. Huang C, Wang Y, Li X, et al. Clinical features of patients infected with 2019 novel coronavirus in Wuhan, China. Lancet. 2020;395 (10223):497-506. doi:10.1016/S0140-6736(20)30183-5

3. Mao L, Jin H, Wang M, et al. Neurologic manifestations of hospitalized patients with coronavirus disease 2019 in Wuhan, China. JAMA Neurol. 2020;77:683-690. doi:10.1001/jamaneurol.2020.1127

4. Oxley TJ, Mocco J, Majidi S, et al. Large-vessel stroke as a presenting feature of COVID-19 in the young. $N$ Engl $\mathrm{J} \mathrm{Med}$. 2020;382:e60. doi:10.1056/NEJMc2009787

5. Belani P, Schefflein J, Kihira S, et al. COVID-19 is an independent risk factor for acute ischemic stroke. Am $J$ Neuroradiol. 2020;41:1361-1364. doi:10.3174/ajnr.A6650

6. Klok F, Kruip M, van der Meer N, et al. Incidence of thrombotic complications in critically Ill ICU patients with COVID-19. Thromb Res. 2020;191:145-147. doi:10.1016/j.thromres.2020.04.013

7. Cui S, Chen S, Li X, Liu S, Wang F. Prevalence of venous thromboembolism in patients with severe novel coronavirus pneumonia. J Thromb Haemost. 2020;18:1421-1424. doi:10.1111/jth.14830

8. Yaghi S, Ishida $\mathrm{K}$, Torres $\mathrm{J}$, et al. SARS-CoV-2 and stroke in a New York healthcare system. Stroke. 2020;51:2002-2011. doi:10.1161/STROKEAHA.120.030335

9. Li Y, Wang M, Zhou Y, et al. Acute cerebrovascular disease following COVID-19: a single center, retrospective, observational study. Stroke Vasc Neurol. 2020;5:e000431. doi:10.1136/svn-2020-000431

10. Terpos E, Ntanasis-Stathopoulos I, Elalamy I. Hematological findings and complications of COVID-19. Am J Hematol. 2020;95:834-847. doi:10.1002/ajh.25829 
11. Varga Z, Flammer AJ, Steiger P, et al. Endothelial cell infection and endotheliitis in COVID-19. Lancet. 2020;395(10234):1417-1418. doi:10.1016/S0140-6736(20)30937-5

12. Avulaa A, Nalleballeb K, Narulaa N, et al. COVID-19 presenting as stroke. Brain Behav Immun. 2020;87:115-119. doi:10.1016/j. bbi.2020.04.077

13. Khan M, Ibrahim RH, Siddiqi SA, et al. COVID-19 and acute ischemic stroke - a case series from Dubai, UAE. Int $J$ Stroke. 2020;15(6):699-700. doi:10.1177/1747493020938285

14. Mowla A, Sizdahkhani S, Sharifian-Dorche M, et al. Unusual pattern of arterial macrothrombosis causing stroke in a young adult recovered from COVID. J Stroke Cerebrovasc Dis. 2020;29(12):105353. doi:10.1016/j.jstrokecerebrovasdis.2020.105353

15. Crippa S, Kägi G, Graf L, Meyer Sauteur PM, Kohler P. Stroke in a young adult with mild COVID-19 suggesting endotheliitis. $N$ Microbes $N$ Infect. 2020;38:100781. doi:10.1016/j. nmni.2020.100781
16. Owolabi L, Raafat A, Enwere O, Mustapha A, Adamu B, AlGhamdi M. Hemorrhagic infarctive stroke in COVID-19 patients: report of two cases and review of the literature. J Commun Hosp Intern Med Perspect. 2021;11(3):322-326. doi:10.1080/20009666.2021.1883814

17. Khedr E, Soliman R, Abo-Elfetof N, et al. Clinical and radiological characteristics of acute cerebrovascular diseases among Egyptian patients with COVID-19 in upper Egypt. Front Neurol. 2021;12:635856. doi:10.3389/fneur.2021.635856

18. Cappellari M, Zini A, Sangalli D, et al. Thrombolysis and bridging therapy in patients with acute ischaemic stroke and Covid-19. Eur J Neurol. 2020;27(12):2641-2645. doi:10.1111/ene.14511

19. Fuentes B, Alonso de Leciñana M, García-Madrona S, et al. Stroke acute management and outcomes during the COVID-19 outbreak: a cohort study from the Madrid stroke network. Stroke. 2021;52(2):552-562.

20. Escalard S, Maïer B, Redjem H, et al. Treatment of acute ischemic stroke due to large vessel occlusion with COVID-19: experience from Paris. Stroke. 2020;51(8):2540-2543. doi:10.1161/STROKEAHA.120.030574

\section{Publish your work in this journal}

The International Medical Case Reports Journal is an international, peer-reviewed open-access journal publishing original case reports from all medical specialties. Previously unpublished medical posters are also accepted relating to any area of clinical or preclinical science. Submissions should not normally exceed 2,000 words or 4 published pages including figures, diagrams and references. The manuscript management system is completely online and includes a very quick and fair peer-review system, which is all easy to use. Visit http://www.dovepress.com/testimonials.php to read real quotes from published authors. 